\title{
Clocking Enhanced Ionization of Hydrogen Molecules with Rotational Wave Packets
}

\author{
Yonghao Mi® ${ }^{1,2, *}$ Peng Peng, ${ }^{2}$ Nicolas Camus, ${ }^{1}$ Xufei Sun,,${ }^{1}$ Patrick Fross, ${ }^{1}$ Denhi Martinez $\odot,{ }^{1}$ Zack Dube, ${ }^{2}$ \\ P. B. Corkum, ${ }^{2}$ D. M. Villeneuve $\odot,{ }^{2}$ André Staudte, ${ }^{2, \dagger}$ Robert Moshammer, ${ }^{1, \ddagger}$ and Thomas Pfeifer ${ }^{1, \S}$ \\ ${ }^{1}$ Max-Planck-Institut für Kernphysik, Saupfercheckweg 1, 69117 Heidelberg, Germany \\ ${ }^{2}$ Joint Attosecond Science Laboratory, National Research Council and University of Ottawa, Ottawa, Ontario KlA OR6, Canada
}

(Received 26 May 2020; revised 14 September 2020; accepted 24 September 2020; published 23 October 2020)

\begin{abstract}
Laser-induced rotational wave packets of $\mathrm{H}_{2}$ and $\mathrm{D}_{2}$ molecules were experimentally measured in real time by using two sequential 25 -fs laser pulses and a reaction microscope. By measuring the timedependent yields of the above-threshold dissociation and the enhanced ionization of the molecule, we observed a few-femtosecond time delay between the two dissociation channels for both $\mathrm{H}_{2}$ and $\mathrm{D}_{2}$. The delay was interpreted and reproduced by a classical model that considers enhanced ionization and thus additional interaction within the laser pulse. We demonstrate that by accurately measuring the phase of the rotational wave packet in hydrogen molecules we can resolve dissociation dynamics which is occurring within a fraction of a molecular rotation. Such a rotational clock is a general concept applicable to sequential fragmentation processes in other molecules.
\end{abstract}

DOI: 10.1103/PhysRevLett.125.173201

Intense light can couple nuclear and electron dynamics leading to hallmark phenomena of molecular strong-field physics such as correlated electron-nuclear energy sharing [1-3], bond softening [4], bond hardening [5], abovethreshold dissociation [2,6,7], light-induced conical and nonconical intersections [8,9], and enhanced ionization $[10,11]$, all of which have been extensively studied in the hydrogen molecule $\mathrm{H}_{2}$ (see, e.g., Refs. [12-15], and references therein). However, since the nuclear dynamics of $\mathrm{H}_{2}$ and its ion proceeds rapidly on a few-femtosecond timescale, it has been a challenge to disentangle the precise timings of the different pathways. Using electron and ion momentum imaging, the bound state wave packet of the $\mathrm{H}_{2}{ }^{+}$molecular ion was recently resolved within the first few femtoseconds [16]. The time delay between abovethreshold dissociation (ATD) and bond softening was determined using polarization-skewed laser pulses [17]. However, the timing of enhanced ionization $[10,11]$ has been difficult to resolve [18-20].

In this Letter, we demonstrate that rotational wave packets of $\mathrm{H}_{2}$ and $\mathrm{D}_{2}$ can serve as an ultrafast clock for probing time delays of the molecular dissociation dynamics on the few-femtosecond timescale within a single multicycle pulse. Using a reaction microscope [21], timeresolved yields of all strong-field dissociation channels

Published by the American Physical Society under the terms of the Creative Commons Attribution 4.0 International license. Further distribution of this work must maintain attribution to the author(s) and the published article's title, journal citation, and DOI. Open access publication funded by the Max Planck Society. were measured, including their relative timing. The difference in their timing is understood and reproduced by a classical model that considers specific triggering times for the different dissociation channels.

As shown in Fig. 1, when $\mathrm{H}_{2}$ is exposed to an intense near-infrared (NIR) laser field, the following steps may occur sequentially. (1) The molecule is singly ionized by absorption of more than 10 NIR photons and a bound molecular ion is created (bound ionization, BI). (2) The bond between the two nuclei stretches, and the molecule absorbs three additional photons at the internuclear distance $R_{1}$ and dissociates along the $2 p \sigma_{u}$ state. (3) The molecule emits one photon at $R_{2}$ and breaks up into a singly charged ion and a neutral atom, via ATD [net two-photon pathway, shown by blue arrows in Fig. 1(a)]. Alternatively, after step (1), the molecule may expand along the $1 s \sigma_{g}$ curve and promote to the $2 p \sigma_{u}$ state by absorbing one additional photon at $R_{2}$ for direct dissociation. This pathway is known as bond softening, which is shown by red arrows in Fig. 1(a). (4) As the bond further stretches, the remaining electron is removed via charge-resonance enhanced ionization (EI) in the strong laser field at a range of critical internuclear distances [10], leading to a Coulomb explosion of the bare protons. Bond softening and ATD are distinguishable from EI by the number of produced protons, as well as in the energy spectrum, as they gain different kinetic energies along their respective dissociative pathways; see Fig. 1(b). For each reaction pathway the sequence of these steps with relative timings $t_{0}, t_{1}, t_{2}$, and (in the case of EI) $t_{3}$ is well defined $[17,18,22,23]$. However, when all processes occur within a single pulse, how can one extract the relative timing between these processes? To answer this question, we design a pump-probe experiment in which the pump 
(a)

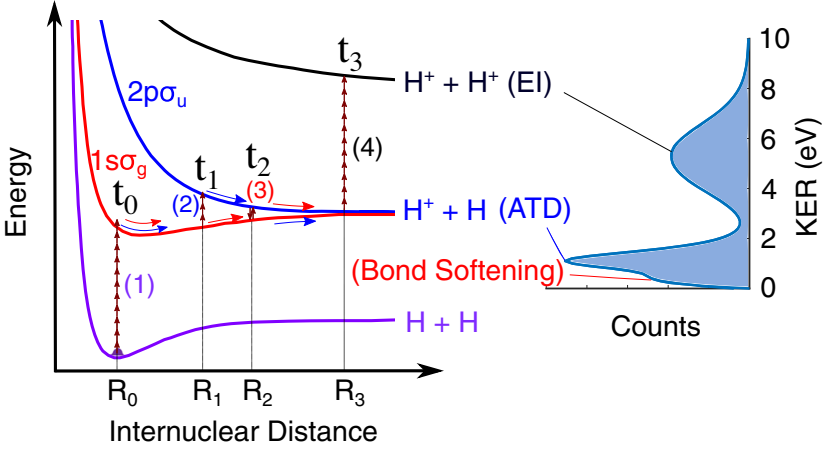

FIG. 1. (a) Sketch of the potential-energy curve of the $\mathrm{H}_{2}$ molecule (see text for details). (b) Kinetic-energy release (KER) spectrum for $\mathrm{H}^{+}$.

pulse only creates rotational wave packets triggering a rotational clock in $\mathrm{H}_{2}$, and the probe pulse ionizes and dissociates the neutral molecule.

Experimentally, 25-fs linearly polarized laser pulses at the central wavelength of $800 \mathrm{~nm}$ are generated by an amplified Ti:sapphire laser. A Mach-Zehnder interferometer is employed to achieve the pump-probe measurement with two 800-nm laser pulses. The pump and probe pulses are focused inside the reaction microscope where they intersect a supersonic $\mathrm{H}_{2}$ or $\mathrm{D}_{2}$ gas jet. The 3D momenta of ions are reconstructed by measuring their time of flight and impact positions in coincidence, which allows distinguishing different ionization channels of the molecules [24]. The intensities of the pump and the probe pulses are estimated at $1 \times 10^{14}$ and $4 \times 10^{14} \mathrm{~W} / \mathrm{cm}^{2}$ for $\mathrm{H}_{2}$ and $1 \times 10^{14}$ and $6 \times$ $10^{14} \mathrm{~W} / \mathrm{cm}^{2}$ for $\mathrm{D}_{2}$. The high probe pulse intensities ensure that the net-two-photon pathway dominates the dissociation process. Thus, the contribution of the bond softening (one-photon) pathway to the enhanced ionization channel can be ignored. In both measurements, the pump pulse is polarized along the gas jet ( $y$ axis), while the probe pulse is polarized along the spectrometer axis ( $z$ axis), as shown in Fig. 2(a). This orthogonally polarized pumpprobe scheme ensures that the dissociation events induced by the pump pulses can be separated from those induced by the probe.

Figure 2(b) shows the kinetic-energy release (KER) distribution as a function of the time delay between the pump and the probe pulses. Here, we calculated the KER as twice the energy of the first detected proton. The data are plotted for fragments within a $45^{\circ}$ cone around the polarization axis of the probe pulse. The peaks of the KER centered at 1.1 and $5.4 \mathrm{eV}$ correspond to the ATD and EI processes, respectively. Distinct delay-dependent oscillation structures in both channels are observed. The timeresolved yields of ATD (blue circles) and EI (red squares) have been extracted and plotted in Fig. 2(c). In order to determine the oscillation frequencies for the two channels, a Fourier transform was performed on their time-dependent traces, shown for ATD (blue) and EI (red) in Fig. 2(d). Two main frequencies, 10.6 and $17.6 \mathrm{THz}$, were observed for both channels, which agree well with the rotational transitions of $\mathrm{H}_{2}, J=0 \rightarrow 2$ and $J=1 \rightarrow 3$, respectively. This confirms that the rotational wave packets were created in the $\mathrm{H}_{2}$ molecule by the pump pulse. As both ATD and EI are sensitive to the molecular alignment, their time-resolved yields reflect the rotational wave packets of $\mathrm{H}_{2}$. It is worth mentioning that the 25-fs pulses are ideal to align $\mathrm{H}_{2}$ compared to few-cycle pulses. This is because the pulse is still short enough that the neighboring rotational states of the molecule can be coherently superimposed, meanwhile the pulse is not so short as to create vibrational wave packets of $\mathrm{H}_{2}{ }^{+}$on top of the rotational wave packets of $\mathrm{H}_{2}$ [25].

Although ATD and EI show identical oscillation structures, which were mapped by the rotational wave packets in the hydrogen molecules, a clear time delay in their traces was observed, as is visible in Fig. 2(c). In order to extract this time delay, we fit their yields $Y_{\mathrm{ATD}}$ and $Y_{\mathrm{EI}}$ by the superposition of $n$ cosine functions as follows:

$$
\begin{gathered}
Y_{\mathrm{ATD}}(t)=\sum_{i=1}^{n} a_{i} \cos \left(\omega_{i} t+\phi_{i}\right)+b_{0}, \\
Y_{\mathrm{EI}}(t)=\sum_{i=1}^{n} c_{i} \cos \left[\omega_{i}(t-\delta t)+\phi_{i}\right]+d_{0},
\end{gathered}
$$

where $\omega_{i}$ is the $i$ th rotational transition angular frequency obtained in the Fourier transform and $n$ is the total number of these frequencies. In the measurement of $\mathrm{H}_{2}, n=2$. The coefficients $a_{i}$ and $c_{i}$ denote the amplitudes of the $i$ th transition frequency of ATD and EI, and $b_{0}$ and $d_{0}$ represent the mean values of the yields of ATD and EI, respectively. $\phi_{i}$ is the phase of the $i$ th frequency of ATD, and $\delta t$ is the extracted time delay of EI with respect to ATD. The fit results of ATD and EI for $\mathrm{H}_{2}$ are plotted in Fig. 2(c). Figure 2(e) shows the same curves as in Fig. 2(c) for a shorter time window between 250 and $350 \mathrm{fs}$. The delay from the fit is $\delta t=5.5 \pm 0.7 \mathrm{fs}$.

How can this time delay be explained? As shown in Fig. 1(a), for enhanced (double) ionization of $\mathrm{H}_{2}$, four sequential steps need to occur in the probe pulse after the creation of rotational wave packets in $\mathrm{H}_{2}$. Under the high intensity of the probe pulse, $\mathrm{BI}$ is almost isotropic in the molecular frame [26], and the ionization yield barely depends on the degree of alignment $\left\langle\cos ^{2} \theta\right\rangle$, where $\theta$ is the angle between the molecular axis and the polarization of the probe pulse. As the dissociation process involves the $1 s \sigma_{g}$ and the $2 p \sigma_{u}$ states, which are coupled through a parallel transition, both ATD and EI are alignmentdependent processes [27,28]. Therefore, the yields of ATD and EI oscillate with increasing pump-probe delay. Figure 3(a) illustrates the intensity distribution of pump and probe pulses as a function of time. We define the time when 
(a)
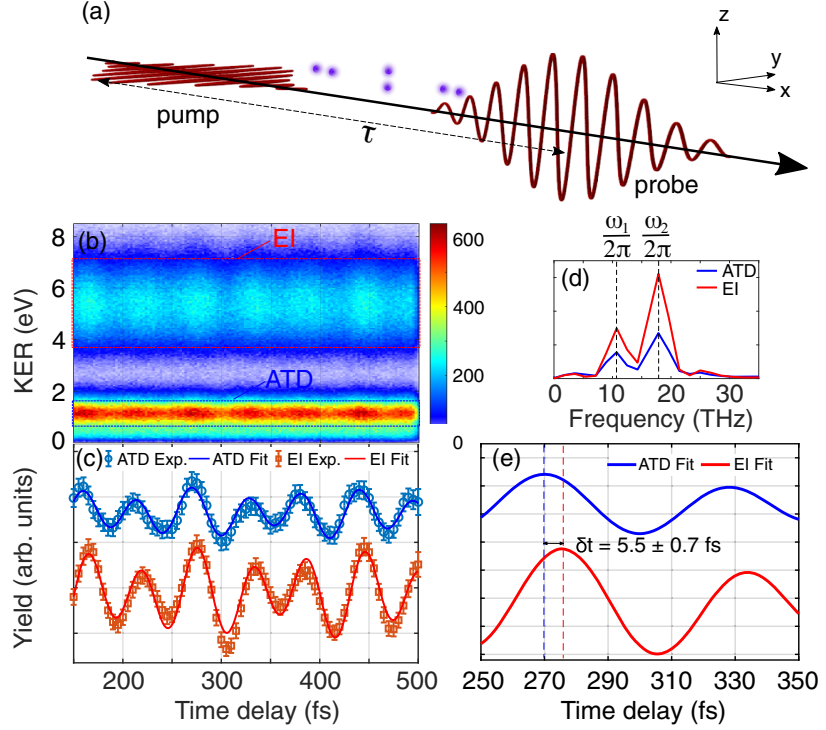

FIG. 2. (a) Sketch of the experiment. The pump pulse creates rotational wave packets in $\mathrm{H}_{2}$ and the probe pulse ionizes and dissociates the molecule. (b) Kinetic-energy release distribution of $\mathrm{H}^{+}$with the condition along the polarization direction of the probe pulse as a function of the pump-probe delay. (c) Timeresolved yields of ATD (blue circle) and EI (red squares). Fit functions of ATD (blue curve) and EI (red curve) were plotted together with the experimental data. (d) Fourier transform of the time-resolved yields of ATD (blue curve) and EI (red curve). (e) Enlargement of the fitted curves of ATD and EI for delays ranging from 250 to $350 \mathrm{fs}$.

the first electron is ionized as $t_{0}$, and the three-photon absorption, the one-photon emission, and the enhanced ionization as $t_{1}, t_{2}$, and $t_{3}$, respectively. Here, $t_{0}$ can be any time in the duration of the probe pulse as long as the intensity at $t_{0}$ is sufficient for removing the first electron. However, $t_{2}-t_{0}$ and $t_{3}-t_{0}$ are quantities which indicate the actual time taken by the molecule to stretch to bond lengths $R_{2}$ and $R_{3}$ that are associated with ATD and EI, respectively, as shown in Fig. 3(a). These quantities remain approximately constant regardless of the pump-probe delay. For EI the intensity required for the second ionization is higher than compared to the net two-photon absorption of the ATD channel. Therefore, the $t_{0}$ of EI can be different than that of ATD. Since the period of the rotational transition $J=1 \rightarrow 3$ of $\mathrm{H}_{2}$ is comparable to the duration of the probe laser pulse, even a few-femtosecond change across the pulse leads to a non-negligible variation of the degree of alignment. Because ATD and EI occur at different times after the first ionization step in the probe pulse, their alignment dependence translates into a temporal shift of their yields as a function of time delay.

Having understood the qualitative origin of the time delay, we now develop a model to calculate the timedependent yields of ATD and EI within the probe pulse. Note that Fig. 3(a) only shows the case in which the final step of EI occurs at a specific time $\tau+t$, where $\tau$ is the pump-probe delay, and $t$ is the time interval from the peak of the probe pulse to the time when EI occurs. To calculate the yields of ATD and EI at the time delay $\tau$, in our model, we calculate the probabilities of ATD and EI for each bin of time and integrate them over the whole pulse envelope. As both ATD and EI stem from BI, the time-dependent ionization rate from the first step $r_{\mathrm{BI}}(\tau)$ is calculated. In this calculation, we assume that the ionization is isotropic (not alignment dependent) and the atomic Ammosov-DeloneKrainov (ADK) model [29] can thus be applied for single ionization of $\mathrm{H}_{2}$. ATD consists of the three-photon absorption (at $t_{1}$ ) and the one-photon emission (at $t_{2}$ ). Therefore, the rate of ATD is given by the product of the three-photon up-transition rate and the one-photon down-transition rate [17]. The $n$-photon transition rate is proportional to $I^{n}(\tau)\left\langle\cos ^{2 n} \theta\right\rangle(\tau)$, where $I$ is the laser intensity and $\left\langle\cos ^{2} \theta\right\rangle$ is the degree of alignment. Modeling EI is challenging due to the complex charge-resonance enhanced ionization mechanism [19,20,30,31]. For simplicity, we treat EI as a multiphoton process. We can thus derive the rates of ATD and EI at times $t_{2}$ and $t_{3}, r_{\mathrm{ATD}}\left(t_{2}\right)$ and $r_{\mathrm{EI}}\left(t_{3}\right)$, as follows:

$$
\begin{gathered}
r_{\mathrm{ATD}}\left(t_{2}\right) \propto r_{\mathrm{BI}}\left(t_{0}\right) I^{3}\left(t_{1}\right)\left\langle\cos ^{6} \theta\right\rangle\left(t_{1}\right) I\left(t_{2}\right)\left\langle\cos ^{2} \theta\right\rangle\left(t_{2}\right), \\
r_{\mathrm{EI}}\left(t_{3}\right) \propto r_{\mathrm{ATD}}\left(t_{2}\right) I^{n}\left(t_{3}\right)\left\langle\cos ^{2 n} \theta\right\rangle\left(t_{3}\right),
\end{gathered}
$$

where $\quad t_{0}=\tau+t-\Delta t_{23}-\Delta t_{12}-\Delta t_{01}, \quad t_{1}=\tau+t-$ $\Delta t_{23}-\Delta t_{12}, t_{2}=\tau+t-\Delta t_{23}$, and $t_{3}=\tau+t$.

Integrating over $t$, the yields of ATD and EI as a function of the pump-probe delay are

$$
\begin{gathered}
\bar{Y}_{\mathrm{ATD}}(\tau)=\int_{-a}^{a} r_{\mathrm{ATD}}\left(\tau+t-\Delta t_{23}\right) d t, \\
\bar{Y}_{\mathrm{EI}}(\tau)=\int_{-a}^{a} r_{\mathrm{EI}}(\tau+t) d t .
\end{gathered}
$$

In the above equations, $n$ is the number of photons absorbed in the enhanced ionization. Inferred from the potentialenergy curve of $\mathrm{H}_{2}$ and the photon energy of the laser, $n=11$. $a$ is a parameter which determines the width of the integration. In this calculation, $a$ was set to $50 \mathrm{fs}$, which ensures that the whole 25-fs pulse was covered. $\left\langle\cos ^{2} \theta\right\rangle$ is calculated by solving the time-dependent Schrödinger equation for $\mathrm{H}_{2}$, which is plotted in Fig. 3(b). $\Delta t_{23}$ denotes the duration of movement of the nuclei from $R_{2}$, where onephoton emission of ATD is triggered to $R_{3}$, at which enhanced ionization takes place. $\Delta t_{12}$ and $\Delta t_{01}$ are the times of bond stretching from $R_{1}$ to $R_{2}$ and from $R_{0}$ to $R_{1}$, respectively. From the potential-energy curve of $\mathrm{H}_{2}, R_{0}, R_{1}$, and $R_{2}$ are determined to be $1.4,3.3$, and 4.7 a.u. approximately. The internuclear distance $R_{3}$, however, depends on the intensity during the enhanced ionization process [32]. $R_{3}$ can be obtained from the KER in the measurement using the following equation: 


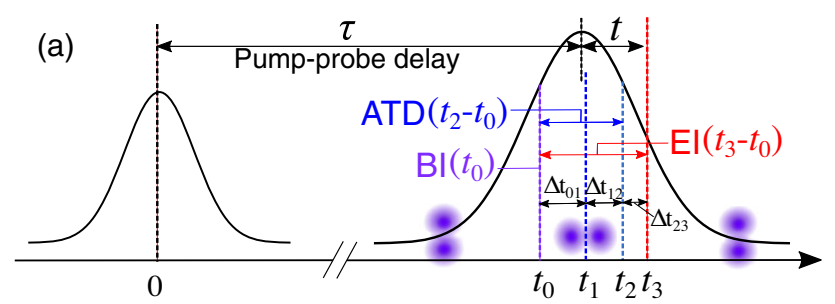

(b)

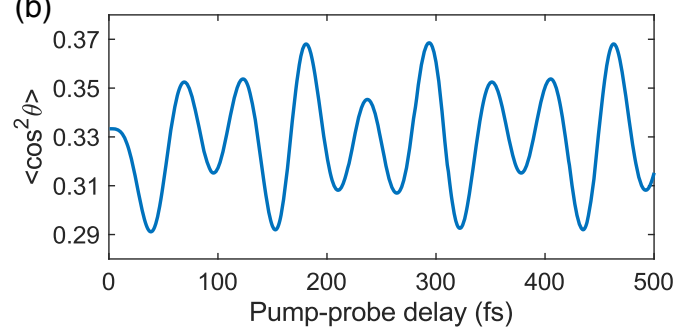

FIG. 3. (a) Sketch of the intensity envelopes of the pump and the probe pulses (see text for details). (b) The calculated degree of alignment of $\mathrm{H}_{2}\left\langle\cos ^{2} \theta\right\rangle$ as a function of the pump-probe delay.

$$
\frac{1}{R_{3}}=E_{\mathrm{EI}}-E_{\mathrm{ATD}}
$$

where $E_{\mathrm{EI}}$ and $E_{\mathrm{ATD}}$ are the KERs of EI and ATD processes, respectively. From the KER spectrum of the $\mathrm{H}_{2}$ measurement, we find that $R_{3}$ is 6.3 a.u.

With the knowledge of $R_{0}, R_{1}, R_{2}$, and $R_{3}$, now the goal is to retrieve the time delays $\Delta t_{01}, \Delta t_{12}$, and $\Delta t_{23}$, and adopt them in the model. We calculated them by simulating the wave packet propagation using the classical model mentioned in Ref. [17]. $\Delta t_{01}, \Delta t_{12}$, and $\Delta t_{23}$ from the simulation are 7.6, 4.1, and $3.2 \mathrm{fs}$, respectively. The calculated yields of ATD (blue curve) and EI (red curve) are shown as a function of the pump-probe delay in Fig. 4(a), together with the experimental data (shaded area given by the error bars). The oscillation structures in the yields of ATD and EI are well reproduced in our calculation. Their yields as a function of the delay from 250 to 350 fs are plotted in Fig. 4(b). A clear delay is observed between the traces of ATD and EI. Using the abovementioned fitting method, the extracted delay is $\delta t=4.9 \mathrm{fs}$, which agrees well with the experiment.

To test the rotational clock method, we performed a measurement on $\mathrm{D}_{2}$ using the same experimental setup and similar pulse intensities. Since the nuclei of $\mathrm{D}_{2}$ are heavier than $\mathrm{H}_{2}$, we expect a longer delay between ATD and EI with the same experimental conditions. The measured timeresolved yields of ATD (blue shaded area) and EI (red shaded area) are shown in Fig. 4(c), and the extracted delay from the experiment is $\delta t=6.5 \pm 0.5 \mathrm{fs}$. The same method of calculation as for $\mathrm{H}_{2}{ }^{+}$was applied to $\mathrm{D}_{2}{ }^{+}$. These results are plotted on top of the experimental data in Fig. 4(c), showing a good agreement with the $\mathrm{D}_{2}{ }^{+}$measurement. Figure 4(d) shows the calculation from 250 to $350 \mathrm{fs}$. The delay between ATD and EI from the calculation is $6.1 \mathrm{fs}$, which also fits the experiment within errors. The modeled delay for $\mathrm{D}_{2}{ }^{+}$is shorter than the modeled delay of $4.9 \mathrm{fs}$ for $\mathrm{H}_{2}{ }^{+}$multiplied by $\sqrt{2}$ to account for the mass difference. This discrepancy has its origin in the combination of pulse envelope and dissociation velocity. Compared to $\mathrm{H}_{2}{ }^{+}$, within the pulse envelope the $\mathrm{D}_{2}{ }^{+}$wave packet samples smaller distances where EI can occur [33]. From our simulation, we find that $\mathrm{D}_{2}{ }^{+}$takes approximately $20 \mathrm{fs}$ to reach a distance of 6.3 a.u. $\left(R_{3}\right.$ of $\left.\mathrm{H}_{2}{ }^{+}\right)$. Hence, within a 25 -fs pulse, $\mathrm{D}_{2}{ }^{+}$propagation is cut short and therefore EI peaks at $R_{3}\left(\mathrm{D}_{2}{ }^{+}\right)=5.6$ a.u., which gives rise to a shorter delay in $\mathrm{D}_{2}{ }^{+}$.

Since the model relies on parameters of the probe pulse, it is natural to consider how the laser field affects the time delay. Therefore, we varied the intensity and pulse duration of the probe pulses, and extracted the time delay for each value. These calculations were performed on $\mathrm{H}_{2}$. As shown in Fig. 4(e), with the pulse duration fixed at $25 \mathrm{fs}$, the delay increases from 4.4 to $5.0 \mathrm{fs}$ as the intensity rises from $1 \times 10^{14}$ to $6 \times 10^{14} \mathrm{~W} / \mathrm{cm}^{2}$. At higher values than $6 \times 10^{14} \mathrm{~W} / \mathrm{cm}^{2}$, the delay stabilized at $5.0 \mathrm{fs}$. Figure 4(f) shows that pulse duration also plays an important role in determining the delay. The delay can be seen to increase from its minimum value of $1.6 \mathrm{fs}$ when a 5 -fs pulse
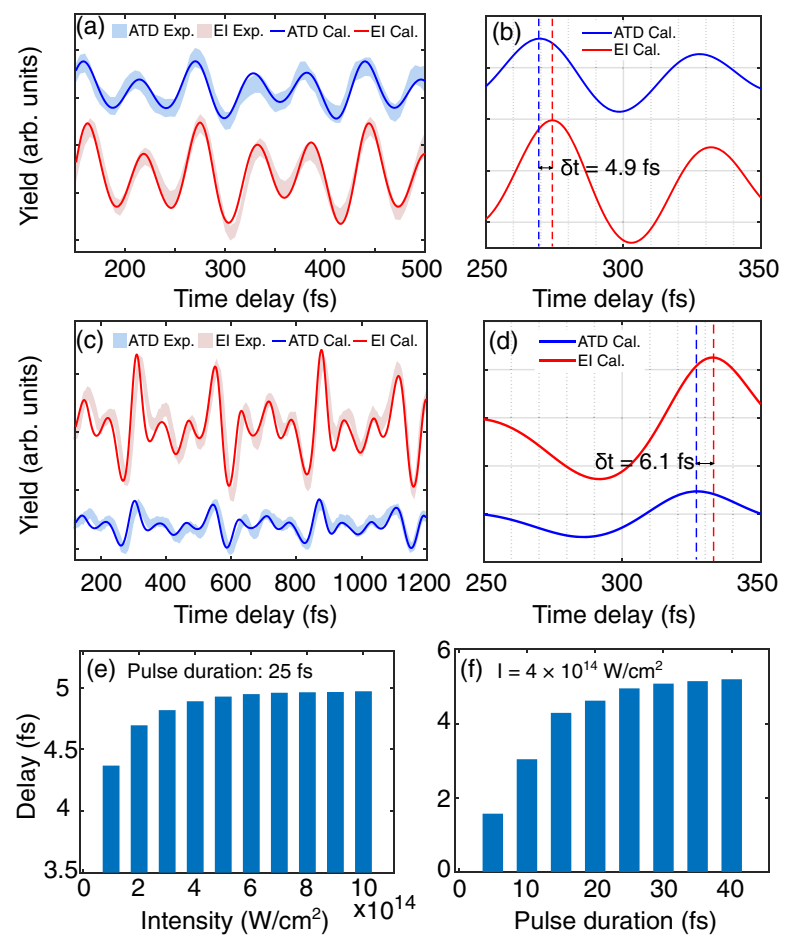

FIG. 4. Calculated yields of ATD (blue curve) and EI (red curve) and experimental data (shaded area given by the error bars) for (a) $\mathrm{H}_{2}$ and (c) $\mathrm{D}_{2}$ as a function of the pump-probe delay. (b), (d) Same as the calculations in (a),(b), but plotted for the same enlarged delays ranging from 250 to 350 fs. (e),(f) Calculated delay $\delta t$ between the yields of ATD and EI for $\mathrm{H}_{2}$ as a function of the intensity and the pulse duration. 
is used to 5.2 fs when a 40 -fs pulse is used. This can be explained by the fact that for a shorter (or less intense) pulse $t_{0}, t_{1}, t_{2}$, and $t_{3}$ are squeezed into a shorter total time range due to the nonlinear dependences of the initial ionization step and the late enhanced ionization step, giving rise to a shorter delay between ATD and EI.

In conclusion, we observed a few-femtosecond delay between the time-dependent yields of ATD and EI of $\mathrm{H}_{2}{ }^{+}$ which were modulated by a rotational wave packet in $\mathrm{H}_{2}$. A model has been developed which reproduces the observed delays well. This method provides the general concept of a molecular rotational clock, which can be extended to other molecules for extracting temporal information of multiple sequential dissociation processes. In combination with a two-color scheme [9], the separation of simultaneously occurring dissociation pathways should become possible. The technique should be applicable to all molecules that can be aligned in a controlled way and preferably dissociated along one of the molecular axes. While for more complex molecules, it may not be possible to identify individual pathways, similar rotational-clock delays of individual fragmentation pathways can still help to discover and separate dissociation mechanisms. In addition, this mechanistic understanding of sequential interactions with a dynamic system also helps in developing general approaches for controlling the ultrafast molecular dynamics.

*yonghao.mi@mpi-hd.mpg.de

†andre.staudte@nrc-cnrc.gc.ca

*robert.moshammer@mpi-hd.mpg.de

sthomas.pfeifer@mpi-hd.mpg.de

[1] J. Wu, M. Kunitski, M. Pitzer, F. Trinter, L. P. H. Schmidt, T. Jahnke, M. Magrakvelidze, C. B. Madsen, L. B. Madsen, U. Thumm, and R. Dörner, Phys. Rev. Lett. 111, 023002 (2013).

[2] P. Lu, J. Wang, H. Li, K. Lin, X. Gong, Q. Song, Q. Ji, W. Zhang, J. Ma, H. Li, H. Zeng, F. He, and J. Wu, Proc. Natl. Acad. Sci. U.S.A. 115, 2049 (2018).

[3] P. Lu, W. Zhang, X. Gong, Q. Song, K. Lin, Q. Ji, J. Ma, F. He, H. Zeng, and J. Wu, Phys. Rev. A 95, 033404 (2017).

[4] P. H. Bucksbaum, A. Zavriyev, H. G. Muller, and D. W. Schumacher, Phys. Rev. Lett. 64, 1883 (1990).

[5] L. J. Frasinski, J. H. Posthumus, J. Plumridge, K. Codling, P. F. Taday, and A. J. Langley, Phys. Rev. Lett. 83, 3625 (1999).

[6] A. Giusti-Suzor, X. He, O. Atabek, and F. H. Mies, Phys. Rev. Lett. 64, 515 (1990).

[7] A. Zavriyev, P. H. Bucksbaum, H. G. Muller, and D. W. Schumacher, Phys. Rev. A 42, 5500 (1990).

[8] A. Natan, M. R. Ware, V. S. Prabhudesai, U. Lev, B. D. Bruner, O. Heber, and P. H. Bucksbaum, Phys. Rev. Lett. 115, 143004 (2016).

[9] M. Kübel, M. Spanner, Z. Dube, A. Y. Naumov, S. Chelkowski, A. D. Bandrauk, M. J. J. Vrakking, P. B. Corkum, D. M. Villeneuve, and A. Staudte, Nat. Commun. 11, 2596 (2020).

[10] T. Zuo and A. D. Bandrauk, Phys. Rev. A 52, R2511 (1995).

[11] E. Constant, H. Stapelfeldt, and P. B. Corkum, Phys. Rev. Lett. 76, 4140 (1996).
[12] A. Giusti-Suzor, F. H. Mies, L. F. DiMauro, E. Charron, and B. Yang, J. Phys. B 28, 309 (1995).

[13] J. H. Posthumus, Rep. Prog. Phys. 67, 623 (2004).

[14] C. Calvert, W. Bryan, W. Newell, and I. Williams, Phys. Rep. 491, 1 (2010).

[15] H. Ibrahim, C. Lefebvre, A. D. Bandrauk, A. Staudte, and F. Légaré, J. Phys. B 51, 042002 (2018).

[16] V. Hanus, S. Kangaparambil, S. Larimian, M. DornerKirchner, X. Xie, M. S. Schöffler, G. G. Paulus, A. Baltuška, A. Staudte, and M. Kitzler-Zeiler, Phys. Rev. Lett. 123, 263201 (2019).

[17] Q. Ji, S. Pan, P. He, J. Wang, P. Lu, H. Li, X. Gong, K. Lin, W. Zhang, J. Ma, H. Li, C. Duan, P. Liu, Y. Bai, R. Li, F. He, and J. Wu, Phys. Rev. Lett. 123, 233202 (2019).

[18] T. Ergler, A. Rudenko, B. Feuerstein, K. Zrost, C. D. Schröter, R. Moshammer, and J. Ullrich, Phys. Rev. Lett. 95, 093001 (2005).

[19] I. Ben-Itzhak, P. Q. Wang, A. M. Sayler, K. D. Carnes, M. Leonard, B. D. Esry, A. S. Alnaser, B. Ulrich, X. M. Tong, I. V. Litvinyuk, C. M. Maharjan, P. Ranitovic, T. Osipov, S. Ghimire, Z. Chang, and C. L. Cocke, Phys. Rev. A 78, 063419 (2008).

[20] H. Xu, F. He, D. Kielpinski, R. T. Sang, and I. V. Litvinyuk, Sci. Rep. 5, 13527 (2015).

[21] J. Ullrich, R. Moshammer, A. Dorn, R. Dörner, L. P. H. Schmidt, and H. Schmidt-Böcking, Rep. Prog. Phys. 66, 1463 (2003).

[22] A. S. Alnaser, B. Ulrich, X. M. Tong, I. V. Litvinyuk, C. M. Maharjan, P. Ranitovic, T. Osipov, R. Ali, S. Ghimire, Z. Chang, C. D. Lin, and C. L. Cocke, Phys. Rev. A 72, 030702(R) (2005).

[23] H. Xu, Z. Li, F. He, X. Wang, A. Atia-Tul-Noor, D. Kielpinski, R. T. Sang, and I. V. Litvinyuk, Nat. Commun. 8, 15849 (2017).

[24] Y. Mi, N. Camus, L. Fechner, M. Laux, R. Moshammer, and T. Pfeifer, Phys. Rev. Lett. 118, 183201 (2017).

[25] A. Rudenko, T. Ergler, B. Feuerstein, K. Zrost, C. Schrter, R. Moshammer, and J. Ullrich, Chem. Phys. 329, 193 (2006).

[26] A. Staudte, S. Patchkovskii, D. Pavičić, H. Akagi, O. Smirnova, D. Zeidler, M. Meckel, D. M. Villeneuve, R. Dörner, M. Y. Ivanov, and P. B. Corkum, Phys. Rev. Lett. 102, 033004 (2009).

[27] K. F. Lee, F. Légaré, D. M. Villeneuve, and P. B. Corkum, J. Phys. B 39, 4081 (2006).

[28] I. A. Bocharova, H. Mashiko, M. Magrakvelidze, D. Ray, P. Ranitovic, C. L. Cocke, and I. V. Litvinyuk, Phys. Rev. A 77, 053407 (2008).

[29] M. V. Ammosov, N. B. Delone, and V. P. Krainov, Sov. Phys. JETP 64, 1191 (1986), http://www.jetp.ac.ru/cgi-bin/ dn/e_064_06_1191.pdf.

[30] G. N. Gibson, M. Li, C. Guo, and J. Neira, Phys. Rev. Lett. 79, 2022 (1997).

[31] A. Saenz, Phys. Rev. A 61, 051402(R) (2000).

[32] P. Peng, S. Peng, H. Hu, N. Li, Y. Bai, P. Liu, H. Xu, R. Li, and Z. Xu, Opt. Express 23, 18763 (2015).

[33] A. Staudte, D. Pavičić, S. Chelkowski, D. Zeidler, M. Meckel, H. Niikura, M. Schöffler, S. Schössler, B. Ulrich, P. P. Rajeev, T. Weber, T. Jahnke, D. M. Villeneuve, A. D. Bandrauk, C. L. Cocke, P. B. Corkum, and R. Dörner, Phys. Rev. Lett. 98, 073003 (2007). 